\title{
THE LOGARITHMIC CENTER OF A PLANAR REGION
}

\author{
DOUGLAS HENSLEY
}

\begin{abstract}
Given a bounded region $S$ in the complex plane, let $f(\beta)$ $=\int_{S} \log |z-\beta| d$ area for $\beta$ any complex number. A logarithmic center of $S$ is an $\alpha$ which minimizes $f(\beta)$. When is $\alpha$ unique?

CONJECTURE. If $S$ is convex then $\alpha$ is unique.
\end{abstract}

THEOREM. If $S$ is convex and symmetric about some line, then $\alpha$ is unique.

0. Introduction. Consider a "reasonable" closed bounded region $S$ in the complex plane.

DEFINITION. A logarithmic center of $S$ is an $\alpha$ which minimizes the logarithmic potential

$$
f_{S}(\beta)=\int_{S} \log |z-\beta| d \text { area } .
$$

The following application to number theory motivates the problem. If $\alpha_{1}$ is a logarithmic center of $S$ and $\alpha_{2}$ is not, then for sufficiently large $x, x\left(S-\alpha_{1}\right)$ will contain more prime Gaussian integers than $x\left(S-\alpha_{2}\right)$. This is seen by comparing the estimate (2) for Gaussian primes in $x\left(S-\alpha_{i}\right)(i=1,2)$.

$$
\operatorname{Li}(S)=\frac{2}{\pi} \int_{S \cap\{z:|z|>2\}} \frac{d \text { area }}{\log |z|} \quad[1],[2] .
$$

For the general region $S$, there need not be a unique logarithmic center. We give two examples.

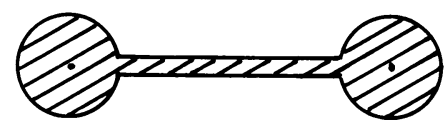

The shaded region has a logarithmic center in each end.

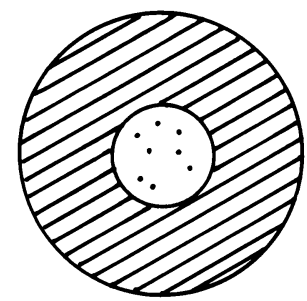

Every point inside the inner of two concentric circles is a logarithmic center of the shaded region.

For certain regions such as a disc, a rectangle, or an equilateral triangle, the symmetry suggests that the geometric center should also be the (unique) logarithmic center.

Received by the editors December 16, 1974.

AMS (MOS) subject classifications (1970). Primary 31 A05, 31 A99, $10 \mathrm{H} 25$.

Copyright $\odot$ 1976, American Mathematical Society 
In $\$ 1$ we give sufficient conditions for a region to have a unique logarithmic center, and state a more general conjecture.

1. Conjecture. If $S$ is convex, $S$ has a unique logarithmic center. ${ }^{1}$

THEOREM 1 . If $S$ is convex and has a line of mirror symmetry, $S$ has a unique logarithmic center.

THEOREM 2. If $S$ is convex, $S_{1}$ and $S_{2}$ concentric disks with radii in ratio 10:11 and $S_{1} \subseteq S \subseteq S_{2}$ then $S$ has a unique logarithmic center.

Proof OF TheOREM 1. We first make several preliminary observations, none of which is at all deep. For the most part, the proofs of these are omitted or drawn sketchily. We shall sometimes write $f$ in place of $f_{S}$. Here $S-\beta$ denotes the translate $S-\beta=\{x-\beta: x \in S\}$.

1 .

$$
\frac{\partial f}{\partial x}(\beta)=-\int_{S-\beta} \frac{x}{x^{2}+y^{2}} d x d y, \text { and } \frac{\partial f}{\partial y}(\beta)=-\int_{S-\beta} \frac{y}{x^{2}+y^{2}} d x d y .
$$

2. Let $\partial S$ be the positively oriented boundary of $S$. Then

$$
\frac{\partial f}{\partial x}(\beta)+i \frac{\partial f}{\partial y}(\beta)=i \int_{\partial S} \log |z-\beta| d z .
$$

REMARK. $\partial f / \partial x+i \partial f / \partial y$ corresponds to the gradient of $f$.

3. Let $g(\beta)=(\partial f / \partial x+i \partial f / \partial y)(\beta)$. Then

$$
\frac{\partial g}{\partial x}-i \frac{\partial g}{\partial y}=-i \int_{\partial S} \frac{d z}{z-\beta}=-i\left[0 \text { if } \beta \notin S, 2 \pi i \text { if } \beta \in S^{0}\right] .
$$

4. If $\beta \notin S, f$ is harmonic at $\beta$. If $\beta \in S^{0},\left(\partial^{2} f / \partial x^{2}+\partial^{2} f / \partial y^{2}\right)(\beta)=2 \pi$. This follows immediately from 3 .

5. If $\alpha$ is a logarithmic center of $S$, and if $c, d \in \mathbf{C}$, then $c \beta+d$ is a logarithmic center of $c S+d$. The same holds for stationary points of $f_{S}$.

6 . There exists at least one logarithmic center of $S$.

Proof. $f(\beta)$ is a continuous function which grows without bound as $|\beta| \rightarrow \infty$. Therefore a minimum exists.

7. Suppose a straight line $M$ passes through a closed convex $S$, dividing $S$ into two parts $S_{1}$ and $S_{2}$. If the reflection of $S_{1}$ across $M$ is a proper subset of $S_{2}$, then no point of $S_{1}$ is a stationary point of $f_{S}$.

Proof. For $\alpha \in S_{1}$, establish coordinate axes through $\alpha$ so that the $y$ axis is parallel to $M$. The $y$ axis divides $S$ into two parts $S_{1}^{\prime}$ and $S_{2}^{\prime}$ so that $S_{1}^{\prime} \subseteq S_{1}$ and $S_{2} \subseteq S_{2}^{\prime}$.

The reflection of $S_{1}{ }^{\prime}$ across the $y$ axis is a proper subset of $S_{2}{ }^{\prime}$, and so

$$
\frac{\partial f s}{\partial x}(\alpha)=-\int_{S} \frac{x}{x^{2}+y^{2}}=-\int_{T} \frac{x}{x^{2}+y^{2}} \neq 0,
$$

where $T=S_{2}{ }^{\prime} \backslash$ the reflection of $S_{1}{ }^{\prime}$, because the integrand is positive and the area of integration nonzero.

1 This is proved in an unpublished manuscript which the author will send to persons requesting it. 
Definition. A saddle point of $f_{S}$ (or "of $f$ " or "of $S$ ") is a stationary point of $f$ which is not a strict local minimum (or maximum, but this is impossible as $f$ is subharmonic).

8. For convex $S$, let $m_{0}$ denote the number of minima of $f_{S}$, and $m_{1}$ the number of saddle points. Then $m_{0}=m_{1}+1$.

REMARK. This is the simplest case of a general theory due to Morse linking the number of maxima, minima, and saddle points to the genus of a surface. See [3].

9. If $\alpha \in S$ is a saddle point of $S$, and if the disc of radius 1 about $\alpha$ is in $S$, then there exists $\beta \in S$ such that $|\alpha-\beta| \geqq e^{\pi / 2}$.

Proof. Establish $x y$ coordinates through $\alpha$ so that $\left(\partial^{2} f / \partial x^{2}\right)(\alpha) \leqq 0$, $\left(\partial^{2} f / \partial x \partial y\right)(\alpha)=0 . f_{S}(\alpha)=f_{(S \backslash \text { disc })}(\alpha)+f_{\text {disc }}(\alpha)$. By fact 4 ,

$$
\left(\partial^{2} / \partial x^{2}+\partial^{2} / \partial y^{2}\right) f_{S \backslash \text { disc }}(\alpha)=0,
$$

and by fact 4 and symmetry (or direct calculation)

$$
\left(\partial^{2} / \partial x^{2}\right) f_{\text {disc }}(\alpha)=\left(\partial^{2} / \partial y^{2}\right) f_{\text {disc }}(\alpha)=\pi .
$$

Now

$$
\frac{\partial^{2}}{\partial x^{2}} f_{S \backslash \text { disc }}(\alpha)=-\int_{S \backslash \text { disc }} \frac{x^{2}-y^{2}}{\left(x^{2}+y^{2}\right)^{2}} d x d y
$$

(this is legitimate if one stays away from the singularity at $\alpha$ ) so

$$
\frac{\partial^{2}}{\partial x^{2}} f_{S}(\alpha)=\pi-\int_{S \backslash \operatorname{disc}} \frac{x^{2}-y^{2}}{\left(x^{2}+y^{2}\right)^{2}} d x d y .
$$

Since $\left(\partial^{2} / \partial x^{2}\right) f_{S}(\alpha) \leqq 0$, we have

$$
\int_{S \backslash \operatorname{disc}} \frac{x^{2}+y^{2}}{\left(x^{2}+y^{2}\right)^{2}} d x d y \geqq \pi .
$$

Converting to polar coordinates, and neglecting the region where $x^{2}-y^{2}$ $<0$, we have

$$
2 \int_{-\pi / 4}^{\pi / 4} \int_{1}^{\partial S} \frac{1}{r} \cos 2 \theta d r d \theta \geqq 4 \Rightarrow \int_{1}^{T} \int_{-\pi / 4}^{\pi / 4} \frac{\cos 2 \theta}{r} \geqq \frac{\pi}{2}
$$

where $T=\sup _{\beta \in S}|\alpha-\beta|$. Thus $\log T \geqq \pi / 2$ and $T \geqq e^{\pi / 2}$ as claimed.

Now suppose $S$ is convex, symmetric about $L$. By 7 and 8 , if $S$ has two logarithmic centers then there exists a saddle point $p$ on $L$, between the two centers, which also lie on $L$. Establish $x y$ coordinates through $p$ with $L$ the $x$ axis. For $g$ above $L$, by the reflection argument of fact 7 with $M$ through $q$ parallel to $L$, we have $(\partial f / \partial y)(q)>0$. Similarly for $q$ below $L,(\partial f / \partial y)(q)<0$. Thus $\left(\partial^{2} / \partial y^{2}\right) f(p) \geqq 0$. By symmetry, or since $\partial f / \partial y \equiv 0$ on $L$, we have $\partial^{2} f / \partial x \partial y \equiv 0$ on $L$. Therefore $\left(\partial^{2} / \partial x^{2}\right) f(p) \leqq 0$, since otherwise $p$ would be a minimum, not a saddle point.

ReMARK. Actually for the symmetric case we only need $p$ on the $x$ axis stationary, with $\partial^{2} f / \partial x^{2} \leqq 0$. 
Now for $(x, y) \in \partial S$ let $\vec{n}(x, y)$ be the unit normal vector outward from the boundary of $S . \vec{n}$ is defined except on a set of measure 0 . Let $\mu(x, y)$ be the $x$ component of $\vec{n}(x, y)$, and $\nu(x, y)$ the $y$ component. Then with a translation of coordinate axes so that $p=0$, we have:

$$
\frac{\partial^{2} f}{\partial x^{2}}(p)=\int_{\partial S} \frac{x \mu(x, y)}{x^{2}+y^{2}} d \text { arc length. }
$$

Assume without loss of generality that the leftmost point of $S$ is at least as far as the rightmost from $p$. Since $(\partial f / \partial x)(p)=0$, the reflection of $(S \cap$ left half plane) across the $y$ axis does not properly contain $(S \cap$ left half plane). Thus for points $q=(x, y)$ of ( $S \cap$ left half plane) whose reflection lies farther to the right than any point of $S$, we have $\mu(q)<0$, and so $x \mu(q) /\left(x^{2}+y^{2}\right)>0$.

Let $a, b$ be respectively the left and right endpoints of $S \cap$ ( $x$ axis). Let $U$ be the portion of $\partial S$ whose $x$ coordinates are between $a$ and $-b$, and let $V=\partial S \backslash U$. Let $y(x)$ be the nonnegative number such that $(x, y(x)) \in \partial S$, for $-b \leqq x \leqq b$.

Then the

$$
\text { integral of } \begin{aligned}
(*) & =\int_{U} \frac{x \mu(x, y)}{x^{2}+y^{2}} d \text { arc length }+\int_{V} \frac{x \mu(x, y)}{x^{2}+y^{2}} d \text { arc length } \\
& \geqq \int_{U} \frac{x \mu(x, y)}{x^{2}+y^{2}} d \text { arc length }+2 \int_{-b}^{b}\left(\frac{-x y^{\prime}(x)}{x^{2}+y^{2}(x)}\right) d x .
\end{aligned}
$$

(Inequality occurs if and only if $V$ has a vertical segment.) As we have noted, the integrand is positive on $U$. The second term

$$
=-2 \int_{0}^{b}\left(\frac{x y^{\prime}(x)}{x^{2}+y^{2}(x)}-\frac{x y^{\prime}(-x)}{x^{2}+y^{2}(-x)}\right) d x .
$$

We shall see that the integrand here is $\leqq 0$, and $=0$ only when $y^{\prime}(x)$ $=y^{\prime}(-x)=0$. Now either (1) $U$ is of positive measure of (2) $y^{\prime}(x)$ is not $\equiv 0$ on $(-b, b)$ or $(3) S$ is a rectangle, and $p$ is the center, hence not a saddle point. In cases $(1)$ or $(2)$, we have $\left(\partial^{2} f / \partial x^{2}\right)(p)>0$, so again $p$ is not a saddle point, a contradiction. We now check that the second integral $\leqq 0$ and thus prove Theorem 1 .

If $y^{\prime}(x) \geqq 0$, then because $S$ is convex, $y(x) \geqq y(-x)$ and $y^{\prime}(-x) \geqq y^{\prime}(x)$, and so the integrand is $\leqq 0\left(<0\right.$ unless $\left.y^{\prime}(x)=y^{\prime}(-x)=0\right)$.

If $y^{\prime}(-x) \leqq 0$, then the same argument shows the integrand again $\leqq 0$.

And if $y^{\prime}(-x) \geqq 0$ and $y^{\prime}(x) \leqq 0$ then both terms of the integrand are $\leqq 0$. This completes the proof of Theorem 1 .

Proof of Theorem 2. We have concentric discs $S_{1}$ and $S_{2}$, of radius 10 and 11 respectively, and $S_{1} \subseteq S \subseteq S_{2} . S$ is assumed convex.

REMARK. One can show there exist radii $m_{1} \neq m_{2}$ such that if $S_{1} \subseteq S \subseteq S_{2}$, $S_{i}$ of radius $m_{i}$ then $S$ has a unique logarithmic center, whether or not $S$ is convex. We do not go into this; the ideas are much the same, with computation taking the place of comparisons by reflection arguments.

Now assume for purposes of contradiction that $f_{S}$ has two minima $\alpha_{1}$ and 
$\alpha_{2}$. Then from 8 there is a saddle point $p$, and from $7, p \in S$.

Case I. Length of $\overline{0} p \geqq 5$, where 0 is the common center of the discs. Construct the line $M$ through $p$ perpendicular to $\overline{0} p$. Let $m_{1}$ and $m_{2}$ be the two points of $\partial S \cap M$. Construct lines $M_{1}$ and $M_{2}$ through $m_{1}, m_{2}$ respectively and tangent to $S_{1}$ as in the figure, at $t_{1}, t_{2}$.

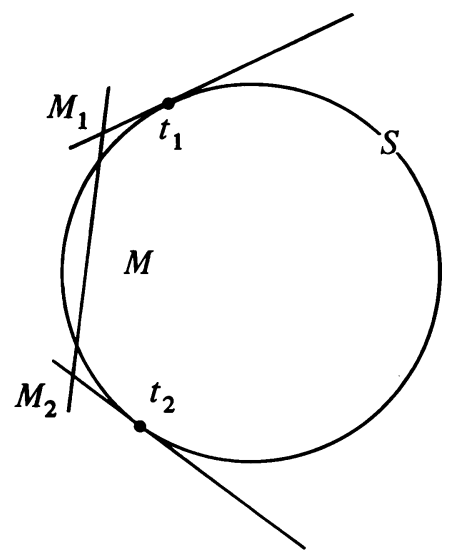

The portion of $S$ to the left of $M$ lies between $M_{1}$ and $M_{2}$, while $S$ contains the quadrilateral $m_{1} m_{2} t_{1} t_{2}$. Since $10^{2}+5^{2} \geqq 11^{2}, M_{1}$ and $M_{2}$ intersect to the left as indicated in the figure, and (left side of $M$ ) $\cap S$ reflects properly into (right side of $M$ ) $\cap S$. But by 7, $p$ cannot even be stationary, a contradiction.

Case II. Length $\overline{0} p<5$. Then $\sup _{\beta \in S}[$ distance $(p, \beta)]<16$, while the disc of radius 5 about $p$ is in $S$. Thus by $9, p$ is not a saddle point since $16<5 e^{\pi / 2}$, a contradiction and Theorem 2 is proved.

\section{REFERENCES}

1. D. Hensley, An asymptotic inequality concerning primes in contours for the case of quadratic number fields, Acta Arith. 28 (1976), 69-79.

2. I. P. Kubilius, The distribution of Gaussian primes in sectors and contours, Leningrad. Gos. Univ. Uč. Zap. 137 Ser. Mat. Nauk 19 (1950), 40-52. (Russian) MR 18, 113.

3. M. Morse and S. S. Cairns, Critical point theory in global analysis and differential topology: An introduction, Pure and Appl. Math., vol. 33, Academic Press, New York and London, 1969, p. 65. MR 39 \#6358.

4. Tibor Radò, Subharmonic functions, Springer, Berlin, 1937.

Department of Mathematics, University of Minnesota, Minneapolis, Minnesota 55455

Current address: Department of Mathematics, University of Illinois, Urbana, Illinois 61801 